\title{
Exertional Rhabdomyolysis: A Case Report of an Exceptionally Elevated Serum Creatine Kinase (CK) Level
}

\author{
${ }^{1}$ Barnet Hospital, Royal Free London NHS Foundation Trust, UK \\ ${ }^{2}$ North Middlesex University Hospital NHS Trust, UK \\ ${ }^{3}$ Guy's and St. Thomas' NHS Foundation Trust, UK
}

Jonathon Kyriakides, BSC, $M B B S^{1^{*}}$, Aria Khani, BSC, MBBS, $M R C P^{2}$ and Rahul Khamar, BSC, $M B B S^{3}$

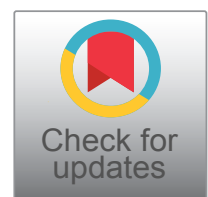

*Corresponding author: Jonathon Kyriakides, BSC, MBBS, Barnet Hospital, Royal Free London NHS Foundation Trust, London EN5 3DJ, United Kingdom, Tel: 020-8216-4600

\begin{abstract}
Serum Creatinine Kinase (CK) is used as a diagnostic and prognostic marker in rhabdomyolysis. We present 32-year-old male with exertional rhabdomyolysis following a spin cycling class, with a peak serum CK level of 332,200 $\mathrm{U} / \mathrm{L}$. He was admitted for intravenous fluid therapy and then followed-up in the outpatient setting; renal function remained stable throughout. We review the literatures and explain why a combination of patient and environmental factors are important in the pathogenesis of exertional rhabdomyolysis. Despite a markedly elevated level of serum $\mathrm{CK}$, and a correlation between serum $\mathrm{CK}$ and the risk of renal dysfunction being well-documented in the literature, normal renal function was noted in our case throughout. Although there is no clear consensus as to whether an elevated serum CK in the absence of renal failure warrants inpatient management, we propose that there could be a cohort of patients who could be managed in the outpatient setting with follow-up.
\end{abstract}

\section{Keywords}

Rhabdomyolysis, Exercise, Creatine kinase, Acute kidney injury, Exercise medicine

\section{Introduction}

Rhabdomyolysis occurs as a result of skeletal muscle damage. A disruption of the integrity of muscle fibres allows release of intracellular skeletal muscle components into the extracellular space and plasma, which can have harmful effects. These components include myoglobin, Creatine Kinase (CK), and electrolytes. High levels of myoglobin in the serum overwhelm the capacity of the myoglobin-binding protein haptoglobin, resulting in myoglobinuria, which can precipitate acute renal failure.

Physical causes of rhabdomyolysis include trauma and exertion, of which strenuous exercise or seizurerelated muscle contractions are common [1]. Nonphysical causes of rhabdomyolysis are more vast; these include a range of medications, toxins, infections, electrolyte abnormalities, endocrine disorders, as well as genetic conditions [1]. Irrespective of cause, membrane ion channel dysfunction and intracellular Adenosine Triphosphate (ATP) depletion allows activation of a final common pathway and a cascade of muscle fibre damage [2].

A triad of myalgia, muscle weakness, and myoglobinuria presenting as dark brown-coloured urine describes the typical features of rhabdomyolysis. However, only $10 \%$ of affected patients have all three symptoms, with dark-coloured urine being seen most often [3]. In fact, rhabdomyolysis can range from an asymptomatic illness with only biochemical disturbance, to a life-threatening condition with electrolyte abnormalities and acute renal failure.

A diagnosis of rhabdomyolysis is made following the detection of intracellular skeletal muscle enzymes in the serum. Myoglobin is rapidly metabolised and excreted, and hence is not suitable as a diagnostic marker. CK is a more sensitive biomarker of skeletal muscle injury. A

Citation: Kyriakides J, Khani A, Khamar R (2021) Exertional Rhabdomyolysis: A Case Report of an Exceptionally Elevated Serum Creatine Kinase (CK) Level. Int J Sports Exerc Med 7:203. doi. org/10.23937/2469-5718/1510203

Accepted: September 28, 2021; Published: September 30, 2021

Copyright: (C) 2021 Kyriakides J, et al. This is an open-access article distributed under the terms of the Creative Commons Attribution License, which permits unrestricted use, distribution, and reproduction in any medium, provided the original author and source are credited. 
Table 1: Blood test results from day 0 (day of presentation) up to day 11 (day of discharge from outpatient setting): $\mathrm{Na}^{+}$(serum sodium; normal range $135-145 \mathrm{mmol} / \mathrm{L}$ ), $\mathrm{K}^{+}$(serum potassium; normal range $3.5-5 \mathrm{mmol} / \mathrm{L}$ ), urea (normal range $1.8-7.1 \mathrm{mmol} / \mathrm{L}$ ), serum creatinine (normal range $60-110 \mu \mathrm{mol} / \mathrm{L}$ ), eGFR (estimated glomerular filtration rate; normal range $<90 \mathrm{ml} / \mathrm{min} / 1.73 \mathrm{~m}{ }^{2}$ ), total bilirubin (normal range < $21 \mu \mathrm{mol} / \mathrm{L}$ ), ALT (alanine aminotransferase; normal range 10-50 U/L), AST (aspartate aminotransferase; normal range $<50 \mathrm{U} / \mathrm{L}$ ), ALP (alkaline phosphatase; normal range $<129 \mathrm{U} / \mathrm{L}$ ), albumin (normal range $35-50 \mathrm{~g} / \mathrm{L}$ ), $\mathrm{CK}(\mathrm{serum}$ creatinine kinase); N/A (not applicable as not performed).

\begin{tabular}{|c|c|c|c|c|c|}
\hline & DAY 0 & DAY 1 & DAY 2 & DAY 4 & DAY 11 \\
\hline $\mathrm{Na}^{+}(\mathrm{mmol} / \mathrm{L})$ & 136 & 140 & 139 & 138 & 139 \\
\hline $\mathrm{K}^{+}(\mathrm{mmol} / \mathrm{L})$ & 4.4 & 4.4 & 4.4 & 4.3 & 4.1 \\
\hline Urea (mmol/L) & 4.1 & 3.1 & 3.1 & 5 & 4.7 \\
\hline Creatinine $(\mu \mathrm{mol} / \mathrm{L})$ & 83 & 73 & 73 & 85 & 79 \\
\hline eGFR (ml/min/1.73 $\left.\mathrm{m}^{2}\right)$ & $>90$ & $>90$ & $>90$ & $>90$ & $>90$ \\
\hline Total bilirubin ( $\mu \mathrm{mol} / \mathrm{L})$ & 8 & N/A & N/A & N/A & 8 \\
\hline ALT (U/L) & 411 & N/A & N/A & N/A & 143 \\
\hline AST (U/L) & 3,529 & N/A & $\mathrm{N} / \mathrm{A}$ & $\mathrm{N} / \mathrm{A}$ & 72 \\
\hline ALP (U/L) & 65 & $\mathrm{~N} / \mathrm{A}$ & N/A & N/A & 63 \\
\hline Albumin (U/L) & 46 & $\mathrm{~N} / \mathrm{A}$ & $\mathrm{N} / \mathrm{A}$ & N/A & 46 \\
\hline CK (U/L) & 332,200 & 129,520 & 103,320 & 22,046 & 485 \\
\hline
\end{tabular}

serum CK level greater than $1,000 \mathrm{U} / \mathrm{L}$ in the presence of skeletal muscle injury is diagnostic for rhabdomyolysis. Serum CK is also a useful prognostic measure for the development of renal impairment, with levels of 5,000 $\mathrm{U} / \mathrm{L}$ or greater being associated with increased risk of Acute Kidney Injury (AKI) [4].

The mainstay of treatment for rhabdomyolysis is supportivewith intravenous fluid resuscitation and correction of serum electrolytes. Intravenous sodium bicarbonate is used to correct systemic acidosis; there is no evidence to support sodium bicarbonate as a superior choice of fluid replacement when acidaemia is not present [5]. Renal replacement therapy may be required.

We presenta case of exercise-induced rhabdomyolysis following a spin cycle class. Biochemical investigations revealed a serum CK level of more than 300,000 U/L, with preserved renal function. In the literature, there is only one case of exercise-induced rhabdomyolysis with a serum CK level greater than that reported in our case study [6].

\section{Case Description}

A 32-year-old male patient presented to the emergency department following a one-day history of passing dark brown-coloured urine. He completed a cycling spin class lasting 45 -minutes three days prior to presentation. He also complained of a two-day history of bilateral quadricep muscle tenderness, worse on walking. He had no other medical problems of note and did not take any prescription or over-the-counter medications. He denied the use of illicit substances. The patient reported an active lifestyle consisting of regular light exercise, including completion of a cycling spin class some months previously.
On initial assessment, the patient was haemodynamically stable and clinically euvolaemic. Initial examination was unremarkable other than mild quadricep muscle swelling bilaterally and bilateral quadriceps muscle tenderness. Neurological examination of the lower limbs was unremarkable, with normal power in all muscle groups bilaterally. Knee extension and flexion against resistance elicited mild discomfort. A urine dipstick on presentation revealed $3+$ blood, 2+ protein, and was negative for nitrites, leukocytes, glucose and ketones.

Blood tests, including a renal and liver profile and serum $\mathrm{CK}$, were performed from the time of presentation up to the point of discharge (Table 1). Blood tests on presentation revealed normal renal function. Serum CK was markedly elevated at 332,200 U/L. A full blood count performed on presentation was unremarkable, with a haemoglobin of $152 \mathrm{mg} / \mathrm{L}$ (normal range 110-150 $\mathrm{g} / \mathrm{L}$ ) and white cell count $8.95 \times 10^{9} / \mathrm{L}$ (normal range 3.5 $11 \times 10^{9} / \mathrm{L}$ ).

The patient was admitted to hospital under the medical team. Oral fluids were encouraged and supplemented with three litres of intravenous fluids in the first 24-hours. 24-hours into the admission, repeat blood tests revealed a serum CK of $129,520 \mathrm{U} / \mathrm{L}$ with normal renal function. Oral fluids were supplemented with a further litre of intravenous fluids in the following 24-hour period.

Serum CK on day 3 of the admission was 103,320 $\mathrm{U} / \mathrm{L}$, and the patient was discharged from the inpatient setting. Bloods tests were repeated two-days postdischarge (day 4 post-presentation) in the outpatient setting: serum CK was 22,046 U/L, renal function remained static.

Bloods testswere repeated one week later (day 11 
post-presentation). Renal function was again normal, and serum aminotransferase levels had drastically fallen. Serum CK was $485 \mathrm{U} / \mathrm{L}$, and the patient was discharged from the outpatient setting.

\section{Discussion}

Exertional rhabdomyolysis refers to the breakdown of skeletal muscle following a period of strenuous activity. Strenuous exercise precipitates both membrane dysfunction as well as intracellular ATP depletion, which propagate a cascade of muscle fibre damage [7]. A study of United States military personnel estimated the incidence of exertional rhabdomyolysis as 22.2 cases per 100,000 per year [8]. Serum CK is used as both a diagnostic marker and prognostic marker in rhabdomyolysis. Hospitalisation is warranted for individuals with significantly raised CK levels or in the presence of complications.

Marathon runners who complete a marathon in a shorter duration have a higher peak of serum CK compared to runners who take longer to complete the same distance [9]. This reveals the importance of the intensity of the exercise, rather than just the length of exercise, for the development of exertional rhabdomyolysis. Environmental factors including temperature and humidity associated with a sauna have been proposed to increase the risk of exertional rhabdomyolysis [10]. The patient in our case study had taken part in a spin class of similar intensity just months previously and experienced only mild muscle aches. This supports the importance of a combination of patient and environmental factors, and not just the length and duration of exercise alone, in the pathogenesis of exertional rhabdomyolysis.

Markedly elevated serum CK levels in exertional rhabdomyolysis have been documented in the literature, for example a 37-year-old male with a peak serum CK level of 95,100 $\mathrm{U} / \mathrm{L}$ following a period of high-intensity training [11]. A case study of 30 patients in a military hospital with exertional rhabdomyolysis revealed that higher levels of serum CK were associated with a prolonged period of hospitalisation [12]. The highest serum CK level measured in these patients was 233,180 $U / L$ [12]. There is only one case in the literature of exertional rhabdomyolysis with a serum CK level higher than that of our case [6].

Renal dysfunction in rhabdomyolysis is owed to a combination of direct myoglobin toxicity, the production of intraluminal casts, and renal vasoconstriction. Although CK is not directly involved in the pathogenesis of renal dysfunction in rhabdomyolysis, serum $\mathrm{CK}$ levels of more than $5,000 \mathrm{U} / \mathrm{L}$ are associated with increased risk of AKI [4]. The patient in our case had a serum creatinine at baseline despite a serum CK level of 332,200 U/L. Similarly, Casares and colleagues report normal renal function in their case of exertional rhabdomyolysis with an exceptionally high serum CK [6]. Despite the serum CK level beingrelated to the degree of muscle damage, we propose that serum CK alone is not always a reliable predictor of renal dysfunction in exertional rhabdomyolysis. Features other than the degree of skeletal muscle injury, such as the presence of medical co-morbidities, are likely to play a key role.

Our patient was admitted to hospital for intravenous fluid hydration because of an exceptionally high serum CK, despite there being no clear consensus as to whether an elevated serum CK in the absence of renal failure warrants inpatient management. Literature suggests to continue intravenous fluids until the serum CK falls below 1,000 U/L and myoglobinuria resolves [13]. However, there are no clear guidelines to support this. In our patient, oral fluid intake was supplemented with intravenous fluids during the first 48-hours of the admission. Renal function remained stable throughout, and serum CK levels were observed to normalise in the outpatient setting over the following week. We propose that there could be a cohort of patients without medical co-morbidities or evidence of renal dysfunction at the time of presentation, who could be managed in the outpatient setting with follow-up. This case study should be combined with other literature when deciding on best management for these patients.

\section{Acknowledgements}

There are no further acknowledgements.

\section{Conflicts of Interest}

The authors declare no conflicts of interest.

\section{Sources of Support}

The authors declare no further sources of support.

\section{Statement of Equal Authors' Contribution}

The authors all contributed to the above manuscript.

\section{References}

1. Bosch X, Poch E, Grau JM (2009) Rhabdomyolysis and acute kidney injury. N Engl J Med 361: 62-72.

2. Torres PA, Helmstetter JA, Kaye AM, Kaye AD (2015) Rhabdomyolysis: Pathogenesis, Diagnosis, and Treatment. Ochsner J 15: 58-69.

3. Cervellin G, Comelli I, Lippi G (2010) Rhabdomyolysis: Historical background, clinical, diagnostic and therapeutic features. Clin Chem Lab Med 48: 749-756.

4. Safari S, Yousefifard M, Hashemi B, Baratloo A, Forouzanfar $M M$, et al. (2016) The value of serum creatine kinase in predicting the risk of rhabdomyolysis-induced acute kidney injury: A systematic review and meta-analysis. Clin Exp Nephrol 20: 153-161.

5. Scharman EJ, Troutman WG (2013) Prevention of kidney injury following rhabdomyolysis: A systematic review. Ann Pharmacother 47: 90-105.

6. Casares P, Marull J (2008) Over a millon Creatine Kinase due to a heavy work-out: A case report. Cases J 1: 173. 
7. Giannoglou GD, Chatzizisis YS, Misirli G (2007) The syndrome of rhabdomyolysis: Pathophysiology and diagnosis. Eur J Intern Med 18: 90-100.

8. Alpers JP, Jones LK (2010) Natural history of exertional rhabdomyolysis: A population-based analysis. Muscle Nerve 42: 487-491.

9. Honda S, Kawasaki T, Kamitani T, Kiyota K (2017) Rhabdomyolysis after High Intensity Resistance Training. Intern Med 56: 1175-1178.

10. Oh RC, Arter JL, Tiglao SM, Larson SL (2015) Exertional rhabdomyolysis: A case series of 30 hospitalized patients. Mil Med 180: 201-207.
11. Siegel AJ, Silverman LM, Lopez RE (1980) Creatine kinase elevations in marathon runners: Relationship to training and competition. Yale J Biol Med 53: 275-279.

12. Schwaber MJ, Liss HP, Steiner I, Brezis M (1994) Hazard of sauna use after strenuous exercise. Ann Intern Med 120: 441-442.

13. Sauret JM, Marinides G, Wang GK (2002) Rhabdomyolysis. Am Fam Physician 65: 907-912. 